\title{
A Neglected Case of Thoracic Aortic Aneurysm- A Case Report
}

\author{
SOHELY SULTANA, ${ }^{1}$ MOHAMMAD FAI SAL I BN KABI R, ${ }^{2}$ TARANAYASMIN, ${ }^{3}$ SHYAMAL KR. ROY, ${ }^{4}$ ASISH SARKAR, ${ }^{5}$ \\ TANJIMA PARVIN ${ }^{6}$
}

\begin{abstract}
:
A 52 year old female reported to surgery department of Bangabandhu Sheikh Mujib Medical University (BSMMU) with dysphasia and exertional chest pain. With detailed history and examination she was diagnosed as a case of epigastric mass and undergone $X$-ray chest. The report revealed enlarged mediastinal shadow with suspicion of thoracic aortic aneurysm. X-ray chest was followed by contrast enhanced CT scan which revealed a large partially thrombosed aneurysmal dilatation of the aortic arch with sharp kinking (bend) at distal end of aneurysm and beginning of descending aorta. Then the patient was referred to cardiac surgery for immediate management. But the patient refused to have a surgery. Then two months later the patient again presented with chest pain and admitted in DMCH. But the patient died.
\end{abstract}

Key words:Thoracic aortic aneurysm (TAA); CT scan.

\section{Introduction:}

Thoracic aortic aneurysm (TAA) is uncommon in people under the age of 60 . About one person in 1000 develops a thoracic aortic aneurysm between the ages of 60 and 65 and this number continues to rise with age. Screening studies show that thoracic aortic aneurysm occurs in 2 to 13 percent of man and 6 percent of women over the age of 65 . However almost 90 percent of aneurysm identified by screening are small (less than $3.5 \mathrm{~cm}$ in diameter) and unlikely to burst. In addition to age, a number of other factors increase a person's risk of developing a thoracic aortic aneurysm are smoking, male gender, some medical conditions such as coronary heart disease, peripheral vascular disease, a family history of aortic aneurysm. ${ }^{1,2}$

Approximately 95\% of aortic aneurysms are caused by atherosclerosis, the narrowing of an artery due to formation of fatty plaque. The muscular middle layer of the artery may be congenitally weak and thus prone to a dissecting aneurysm. High blood pressure (hypertension) intensifies the force of blood on the walls of the arteries and contributes to the development of dissecting aneurysm.

1. Specialist, Dept. of Radiology \& Imaging, United Hospital Ltd.

2. MD Student, Department of Cardiology, BSMMU

3. Assistant Professor, Department of Radioligy \& Imaging, Enam Medical College \& Hospital.

4. Assistant Professor, Department of Radioligy \& Imaging, Sir. Salimullah Medical College \& Hospital.

5. Medical Officer, Department of Radiology \& Imaging, BSMMU.

6. Assistant Professor, Department of Cardiology, BSMMU.

Correspondence : Dr. Sohely Sultana, Specialist, Dept. of Radiology \& Imaging, United Hospital Ltd. e-mail: Ssohely71 @Yahoo.Com. Mobile: 01755517328.
Diseases that are associated with aortic aneurysm are Marfan syndrome,Loeys-Dietz syndrome, Turner's syndrome and Scleroderma. Chest x-rays, computed tomography (CT) scans or ultrasound imaging is used to confirm the presence of an aneurysm. Approximately 15,000 deaths occur each year in the United States due to thoracic aortic aneurysm usually because of rupture. Once rupture has occurred the success rate of surgery is much lower than if surgery is performed electively. Prior to rupture the goal of therapy is to treat the aneurysm before rupture. ${ }^{3}$

Aneurysm repair is the primary treatment for aneurysm that are symptomatic or at a high risk for rupture. Repair however is associated with other risks and complications. The risk of elective repair must be balanced against the risk of complications or death from an untreated aneurysm. This decision requires understanding of the usual course in patients with untreated aneurysm risk of rupture. The risk of rupture of small aneurysms (smaller than $4.0 \mathrm{~cm}$ ) is much less than the risk of rupture of large aneurysms (larger than $6.0 \mathrm{~cm}$ ) In addition to size the risk of thoracic aortic aneurysm rupture depends upon the rate at which the aneurysm is expanding the evidence suggests that aneurysms expand at an average rate of 0.30 to $.4 \mathrm{~cm}$ per year. Aneurysm that expands rapidly are at high risk of rupture. Growth tends to be more rapid in smokers and less rapid in patients with peripheral artery disease or diabetes mellitus. Some aneurysms for unclear reasons remain relatively fixed in size for a period of time and then undergo rapid expansion. The risk of rupture of large aneurysm $(>5.0 \mathrm{~cm})$ is significantly greater is women than man. $2,3,4$

\section{Case Report:}

A 52 year old woman got admitted into cardiology department of BSMMU with the complaints of dysphasia for 1 month 
and exertional chest pain for 2 years. She had history of irregular intake of antihypertensive. She was not diabetic. Excepting for the last 1 month she never experienced resting chest pain. She had no H/O severe chest pain or pain (tearing pain) in the back or any $\mathrm{H} / \mathrm{O}$ collapse or blackout.

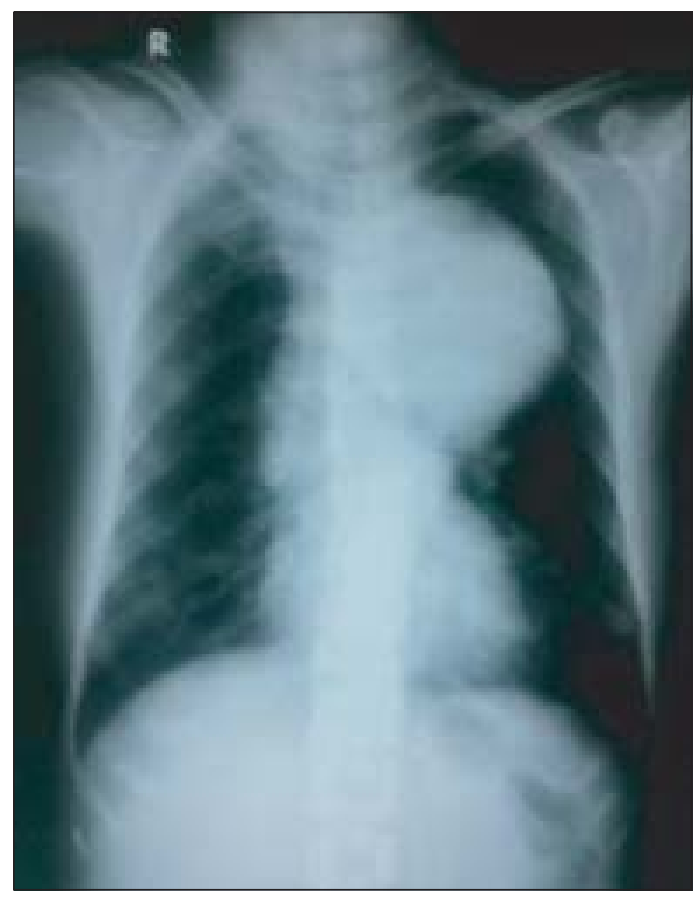

Fig.-1: $X$-ray chest PA view showed enlarged mediastinal shadow and dilatation of the arch and descending aorta.

On admission, the patient was ill-looking and anxious, pulse -92/min with radio-radial delay; decreased volume also found in brachial and axillary artery. BP was 140/80 mm Hg in right armand 120/75 mm Hg in left arm. She had normal jugular venous pressure (JVP). Precordium: S1+S2+O; Suprasternal

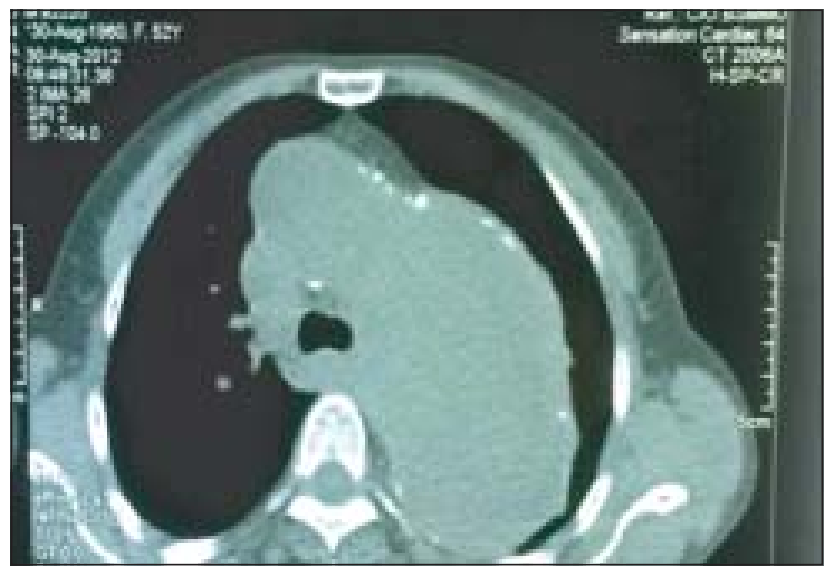

Fig.-2: Non- contrast CT scan revealed a massive $(10.0 \times 8.6 \times 7.5 \mathrm{~cm})$ partially thrombosed aneurysmal dilatation of the aortic arch. pulsation was present. Breath sound was vesicular with no added sound. Routine blood investigations (CBC, S. creatinine, electrolytes, RBS) were unremarkable, ECG: Normal, Echocardiography: Aortic arch was dilated. X-ray chest PA view showed aneurysmal dilatation of the arch and descending aorta. Contrast enhance CT scan revealed

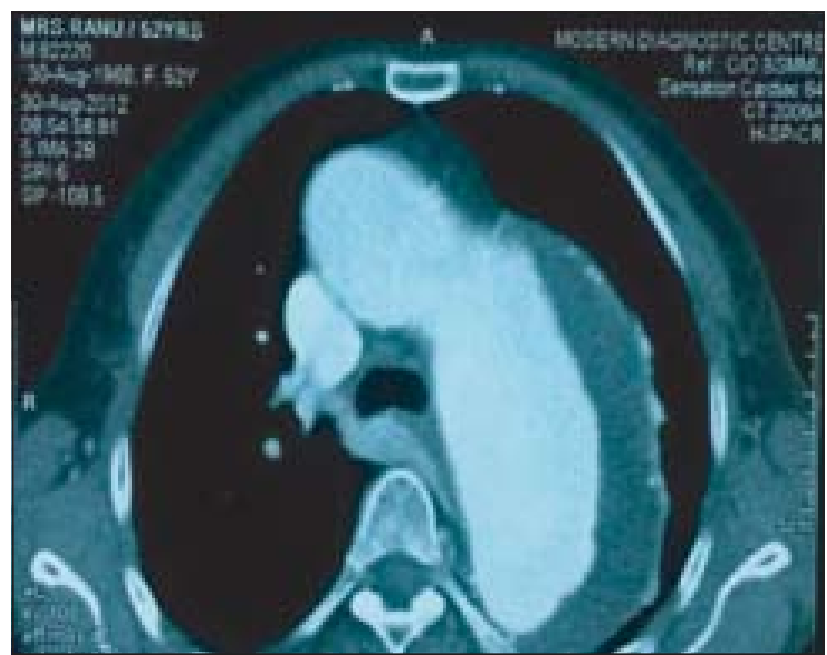

Fig.-3:Cortrast enhanced CT scan revealed a massive $(10.0 \times 8.6 \times 7.5 \mathrm{~cm})$ partially thrombosed aneurysmal dilatation of the aortic arch.

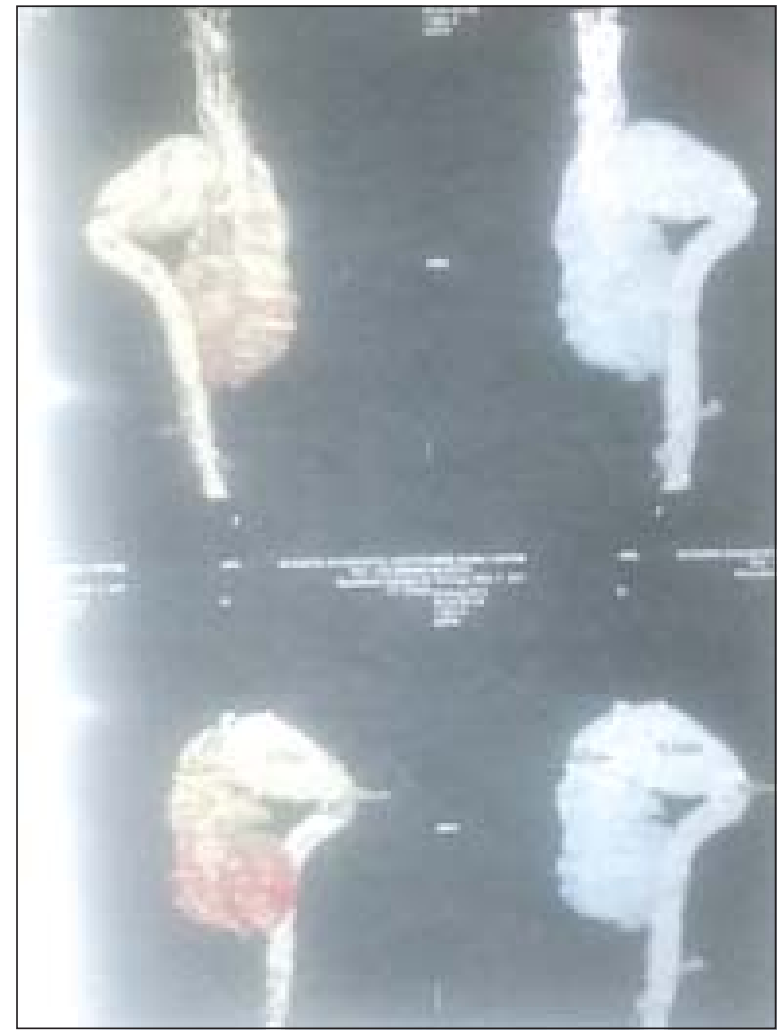

Fig.-4:CT angiogram showing aneurismal dilatation of the aortic arch with sharp kinking (bend) at distal end of aneurysm and beginning of descending aorta. 
massive (10.0x 8.6x $7.5 \mathrm{~cm}$ ) partially thrombosed aneurysmal dilatation of the aortic arch with sharp kinking (bend) at distal end of aneurysm and beginning of descending aorta. CT angiogram reveled a large aneurysmal dilatation of aortic arch measuring $10.0 \times 8.6 \times 7.5 \mathrm{~cm}$ extending up to the beginning of thoracic aorta having large intramural thrombus and wall calcification are noted in the dilated part. Then the patient was referred to cardiac surgery for immediate management. But the patient refused to have surgery. Two months later she again presented with severe agonizing chest pain and was admitted into DMCH. But the patient died.

\section{Discussion:}

Although it was reported that thoracic aortic aneurysm was uncommon in people under the age of 60 and more common in male. ${ }^{1,2}$ It was seen that in present case the patient was of 52 years and was female. It was reported that $95 \%$ of aortic aneurysms are caused by atherosclerosis and hypertension intensifies the force of blood on the walls of the arteries and contributes to the development of dissecting aneurysm. ${ }^{2}$ In present case she had only hypertension (Blood pressure 140/90 mm of Hg). Often, this aneurysm was associated with Marfan syndrome, Loeys-Dietz syndrome, Turner's syndrome and Scleroderma. ${ }^{1,2}$ This present case had no such clinical association.

Chest x-rays, computed tomography (CT) scans or ultrasound imaging is used to confirm the presence of an aneurysm. This current case was presented with mass in mediastinum from thoracic aorta with suspicion of thoracic aortic aneurysm on X-ray chest PA view then followed by contrast enhanced CT scan which revealed a large partially thrombosed aneurysmal dilatation of the aortic arch with sharp kinking (bend) at distal end of aneurysm and beginning of descending aorta. Mortality commonly occurred due to thoracic aortic aneurysm usually because of rupture. Once rupture had occurred the success rate of surgery was much lower than ifsurgery was performed electively. ${ }^{3}$ This woman died due to rupture of the untreated aneurysm. It was observed that risk of rupture of small aneurysms (smaller than $4.0 \mathrm{~cm}$ ) is much less than the risk of rupture of large aneurysms (larger than $6.0 \mathrm{~cm}$ ). The risk of rupture of large aneurysm $(>5.0 \mathrm{~cm})$ was significantly greater is women than man. ${ }^{3,4}$ Similar condition occurred in this current case. The aneurysm was $10.0 \times 8.6 \times 7.5 \mathrm{~cm}$ and the subject was female.

\section{Conclusion:}

Most people with thoracic aortic aneurysm live healthy symptom free lives. The decision to undergo surgery involves weighing the risk of aneurysm rupture versus the risks of surgical procedure. While some general guidelines are suggested based upon the aneurysm size and the rate at which it is enlarging. Each treatment decision should be made on an individual basis. Patients should be discussedabout their individual risk of surgery with an experienced healthcare provider to make an informed decision.

\section{Conflict of interest: None.}

\section{References:}

1. Desforges JF. Thoracic aortic aneurysms.New England J Med 1993; 328(16): 1167-1172.

2. Oureil K, Green RM, Donaayre C, Shortell CK, Elliot J, DeWeere JA. An evaluation of new methods of expressing aortic aneurysm size: Relationship to rupture J Vasc Surg 1992; 15(1): 12-25.

3. Boline PD, Anderson AV. Thoracic aortic aneurysm.AJCM 1989; 2(3): 114-116.

4. Frame PS, Inyleack DG, Patterson C. Screening for thoracic aortic aneurysms in men ages 60-80 years. A caseEffectiveness analysis.Annuals internal Medicine 1993; 119(5): 411-416. 